\title{
ASYMPTOTIC BEHAVIOR OF TWO SEMI-LINEAR ELLIPTIC FREE BOUNDARY PROBLEMS
}

\author{
Thomas I. Vogel
}

Given a bounded open set $\Omega \subseteq \mathbf{R}^{n}$ with $C^{2+\alpha}$ boundary and a monotone increasing function $f(t)$ with $f(0)=0$, this paper treats two related exterior free boundary problems:

Problem A: Given $\lambda>0$, determine $u \in C_{0}^{2+\alpha_{1}}\left(\mathbf{R}^{n}-\Omega\right)$ satisfying:

$$
\begin{aligned}
\Delta u & =\lambda f(u) & & \text { in } \mathbf{R}^{n}-\bar{\Omega} \\
u & =1 & & \text { on } \partial \Omega .
\end{aligned}
$$

Problem B: Given $c>0$, determine $v \in C_{0}^{2+\alpha_{1}}\left(\mathbf{R}^{n}-\Omega\right)$ satisfying:

$$
\begin{aligned}
\Delta v & =f(v) & & \text { in } \mathbf{R}^{n}-\bar{\Omega} \\
v & =c & & \text { on } \partial \Omega .
\end{aligned}
$$

In both problems, the free boundary is the boundary of the support of the sought function.

Problem A comes from the Langmuir-Hinshelwood model for chemical kinetics (among biochemists this is known as Michaelis-Menten kinetics) ([1], [2]). $\Omega$ then represents a patch of constant concentration of a reactant diffusing into a substrate. Problem B has appeared in a paper by Caffarelli and Spruck [3], in which they show that if $\Omega$ is convex, then the level surfaces of $u$ are convex surfaces. A special case of Problem B appears in continuous hot-dip galvanizing ([9]).

Section 2 is concerned about existence and uniqueness for fixed $c$ or $\lambda$. From this point of view, Problem A is contained in Problem B, therefore in this section we deal only with Problem B. The results follow easily from the work that has been done on the interior problem ([5], [7], [8]). With this as a starting point, we attempt to determine characteristics of the free boundary. The main thrust of this paper is to show that in $R^{2}$, as $\lambda \rightarrow 0$ (Problem A) or as $c \rightarrow \infty$ (Problem B), the free boundaries are asymptotic to a family of circles. By this we mean the following. Let $p$ be a point in $\Omega$, let $d(p)$ be the distance from $p$ to the point on the free boundary closest to $p$, and let $d_{1}(p)$ be the distance from $p$ to the point on the free boundary farthest from $p$. Then as $\lambda \rightarrow 0$ (Problem A) or as $c \rightarrow \infty$ (Problem B), the ratio $d_{1}(p) / d(p)$ approaches 1. Thus, if we scale the picture so that the point on the free boundary closest to $p$ is at distance 1 from $p$, then the free boundary in this scaled 
picture approaches a unit circle uniformly. No assumption of regularity is made on the free boundary. This fact has been proven in Problem B for $f(t)=\chi_{\{t>0\}}$ ([9]), where $\chi_{U}$ is the characteristic function of $U$. This is the limiting case as $p \rightarrow 0$ of $f(t)=t^{p}$. It is therefore a natural generalization to consider the problem for more general $f$ 's. The behavior of problem $A$ as $\lambda$ tends to infinity has been analyzed in [5].

If the constants $c$ and $\lambda$ are allowed to vary, then Problems A and B are not the same. Indeed, the only case in which the problems are equivalent is when $f(t)=k t^{p}$ for $t>0$. In that case, a solution to Problem B may be scaled to solve problem A with $\lambda=c^{p-1}$. For general functions $f(t)$ the two problems are distinct. They are similar enough, however, that the methods overlap greatly. Section 3 obtains the asymptotic result for Problem A, and in $\S 4$ the appropriate changes are made to obtain the analogous result for problem B. The argument in $\S 3$ uses radially symmetric solutions for comparisons. The proof uses the concept of asymptotic independence, and gives a partial answer to the question: if a family $\left\{F_{R}(x)\right\}$ is asymptotically independent of $R$ for large $x$, under what conditions will the family of inverse functions $\left\{F_{R}^{-1}(y)\right\}$ be asymptotically independent of $R$ for large $y$ ? One can think of this question in a more concrete fashion: if the asymptotic expansion of $F_{R}(x)$ as $x \rightarrow \infty$ has no $R$ dependence in the highest order term, will this also be true of the inverse function $F_{R}^{-1}(y)$ ? Without futher conditions, the answer is "no", as shown by the example of $\S 3$.

The reason that the methods of the present paper do not extend to $n>2$ is that the integral equation which corresponds to (3.3) clearly has asymptotic dependence on $R$ in higher dimensions.

2. Existence and uniqueness. We will deal with Problem $B$ in this section, since if $\lambda$ and $c$ are fixed, problem $\mathrm{A}$ is a special case of problem B. Let $\Omega \subseteq \mathbf{R}^{n}$ be a bounded open set with $C^{2+\alpha}$ boundary, and let $f(t)$ be a continuous function defined for all $t \in \mathbf{R}$ have the following properties:

(a) $f(t)$ is monotone increasing

(b) $f(t)=0$ for $t \leq 0, f(t)>0$ for $t>0$

(c) $f(t)$ is $C^{1}$ except at $t=0$

(d) $\lim _{t \downarrow 0} \frac{f(t)}{t^{p}}=k$ for some $0<p<1$ and some $0<k<\infty$.

Let $c$ be a positive constant. We will show that the there exists a function $v \in C_{0}^{2+\alpha_{1}}\left(\mathbf{R}^{n}-\Omega\right)$ solving equation (1.2), that is, solving Problem B. 
To do this, we need the following existence lemma.

LEMMA 2.1. Let $U \subseteq \mathbf{R}^{n}$ be a bounded open set with $C^{2+\alpha}$ boundary, let $f(t)$ be as above, and let $\phi(x)$ be a non-negative element of $H^{1}(U)$. Then there exists a unique function $w \in C^{2+\alpha_{1}}(U)$ with $(w-\phi) \in H_{0}^{1}(U)$ which is a classical solution of

$$
\Delta w=f(w) \text { in } U
$$

Here $\alpha_{1}=\min (\alpha, p)$.

Proof. The existence proof is much the same as the analogous result for $f(w)=w^{p}$ in [7]. The existence of a weak solution is shown variationally by minimizing the functional

$$
J(v)=\int_{\Omega}\left(\frac{|\nabla v|^{2}}{2}+F(v)\right) d x, \quad v \in K_{\phi}
$$

where $F(t)=\int_{0}^{t} f(s) d s$, and $K_{\phi}=\left\{v \in H^{1}(\Omega) \mid(v-\phi) \in H_{0}^{1}(\Omega)\right\}$. The argument that this is a classical solution is the same as in [7]. Uniqueness comes from Theorem 9.3 of [6] since $f(t)$ is monotone.

Lemma 2.1 implies uniqueness for the solution of Problem B immediately. Indeed, if $u_{1}$ and $u_{2}$ both solve (1.2) and have compact support then we can let $U=B_{R}-\bar{\Omega}$, where $B_{R}=B_{R}(0)$ is a large enough ball to contain $\operatorname{supp}\left(u_{1}\right) \cup \operatorname{supp}\left(u_{2}\right)$. Then $u_{1}$ and $u_{2}$ will have the same boundary values on $\partial U$, so that we can apply Lemma 2.1.

To prove the existence of a solution to problem $\mathrm{B}$, we must find a solution $w_{R}$ to (2.2) with $U=B_{R}-\bar{\Omega}$ and boundary values $w_{R}=c$ on $\partial \Omega$, $w_{R}=0$ on $\partial B_{R}$, and then show that $R_{1}$ sufficiently large,

$$
\operatorname{supp}\left(w_{R_{1}}\right) \cap \partial B_{R_{1}}=\varnothing .
$$

We will then let $u$ be the extension of $w_{R_{1}}$ by zero.

LemMa 2.2. Suppose $\Omega_{1} \subseteq \Omega_{2} \subseteq B_{R}$. Let $u_{i}, i=1,2$, solve (2.2) with $U_{i}=B_{R}-\bar{\Omega}_{i}$ and with boundary data

$$
\phi_{i}=\left\{\begin{array}{ll}
c_{i} & \text { on } \partial \Omega_{i} \\
0 & \text { on } \partial B_{R}
\end{array} .\right.
$$

Assume $c_{2} \geq c_{1}$. Then $u_{2}(x) \geq u_{1}(x)$.

Proof. Since $u_{1}$ is subharmonic, $u_{1}(x) \leq c_{1} \leq c_{2}$ on $\partial \Omega_{2}$. We may now apply Theorem 9.2 of [6] in $U_{2}$.

Assume for simplicity that $0 \in \Omega$. From the above lemma we may conclude the following: If $\Omega \subseteq B_{R_{1}} \subseteq B_{R}$ and $v$ solves (2.2) with boundary 
data

$$
\phi=\left\{\begin{array}{ll}
c & \text { on } \partial \Omega \\
0 & \text { on } B_{R}
\end{array},\right.
$$

then $v$ is less than the radially symmetric function $w\left(x ; R, R_{1}\right)=$ $w\left(|x| ; R, R_{1}\right)$ which solves (2.2) in $B_{R}-\bar{B}_{R_{1}}$ with boundary values $c$ on $\partial B_{R_{1}}$ and 0 on $B_{R}$. Thus, to show the existence of a solution to (2.2) it suffices to show that given $R_{1}$, there is a value of $R$ sufficiently large to ensure that $\operatorname{supp}\left(w\left(x ; R, R_{1}\right)\right) \cap \partial B_{R}=\varnothing$.

LEMMA 2.3. If $\left(w ; R, R_{1}\right)$ solves:

$$
\begin{aligned}
w^{\prime \prime}+\frac{1}{r} w^{\prime} & =f(w) \quad R_{1}<r<R, \\
w\left(R_{1}\right) & =c \\
w(R) & =0,
\end{aligned}
$$

then for $R$ sufficiently large, $w\left(r ; R, R_{1}\right)=0$ for $R^{*}<r<R$. Here $R^{*}=$ $R^{*}\left(c, R_{1}, f\right)$. (Of course $w^{\prime \prime}+w^{\prime} / r$ is the radial Laplacian.)

Proof. By property (2.1), (d) of $f(t)$, we must have $f(t) \geq b t^{p}$ on $[0, c]$ for some $b>0$. Let

$$
R^{*}=\frac{1}{1-p} \sqrt{\frac{2(1+p)}{b}} c^{(1-p) / 2}+R_{1},
$$

and assume $R>R^{*}$. I will prove that this suffices. Define $x(r)$ by:

$$
x(r)= \begin{cases}(1-p)^{2 /(1-p)}\left(\frac{b}{2(p+1)}\right)^{1 /(1-p)}\left(R^{*}-r\right)^{2 /(1-p)} & r<R^{*} \\ 0 & R^{*} \leq r\end{cases}
$$

One can verify that:

$$
\begin{array}{ll}
x^{\prime \prime}(r)=b x^{p} & \text { on }\left[R_{1}, \infty\right) \\
x\left(R_{1}\right)=c & \\
x^{\prime}(r) \leq 0 & \text { on }\left[R_{1}, \infty\right)
\end{array}
$$

Thus $x^{\prime \prime}(r)+(1 / r) x^{\prime}(r) \leq f(x(r))$ on $\left[R_{1}, \infty\right)$. From Theorem 9.2 of [6], the comparison principle for quasi-linear elliptic operators, $x(r) \geq v(r)$ on $\left[R_{1}, R\right]$. This gives the desired result.

THEOREM 2.4. Given a bounded $\Omega \subseteq \mathbf{R}^{n}$ with $C^{2+\alpha}$ boundary and a function $f(t)$ satisfying properties $(2.1),(a)-(d)$, there is a solution to Problem $B$. 
Proof. Find $R_{1}$ so that $\Omega \subseteq B_{R_{1}}$. Let $w(x)$ solve (2.2) with $U=$ $B_{R^{*}\left(c, R_{1}, f\right)}-\bar{\Omega}$ and boundary values $c$ on $\partial \Omega$ and 0 on $\partial B_{R^{*}\left(c, R_{1}, f\right)}$. From Lemma 2.3, $\operatorname{supp}(w) \cap \partial B_{R^{*}}=\varnothing$. Extend $w(x)$ to the rest of $\mathbf{R}^{n}$ by zero. The result is the desired function $v(x)$.

Definition. The set $\Gamma=\partial\{u>0\}$ is called the free boundary. On $\Gamma$ we have $u=0,|\nabla u|=0$. No regularity claims are made for $\Gamma$. The usual regularity results ([4], Chap. 2) do not apply the case $f(0)=0$.

\section{Asymptotoc behavior of Problem $A$ as $\lambda$ approaches zero.}

LEMma 3.1. Let $\Omega_{1} \subseteq \Omega_{2}$ be bounded open sets and let $\lambda_{1} \geq \lambda_{2}$. Let $u_{i}$ solve Problem $A$ corresponding to $\Omega_{i}$. Then $u_{2}(x) \geq u_{1}(x)$ everywhere.

Proof. We have

$$
\Delta u_{1}-\lambda_{2} f\left(u_{1}\right) \geq \Delta u_{2}-\lambda_{2} f\left(u_{2}\right)=0 .
$$

Moreover, if $\operatorname{supp}\left(u_{1}\right) \cup \operatorname{supp}\left(u_{2}\right) \subseteq B_{R}, u_{1}(x) \leq u_{2}(x)$ on $\partial\left(B_{R}-\bar{\Omega}_{2}\right)$, from the subharmonicity of $u_{1}$. We therefore can apply Theorem 9.2, [6] for the desired result.

As in [9], we will use radial solutions to gain insight into the asymptotic behavior of general solutions. We will see that as $\lambda$ tends to zero, the free boundaries are asymptotic to a family of circles, as in the simpler case considered in [9]. For reasons which will become clear later on, we set $1 / \mu=\lambda$, and we therefore consider the radially symmetric problem:

$$
\begin{gathered}
w^{\prime \prime}(r)+\frac{1}{r} w^{\prime}(r)=\frac{1}{\mu} f(w(r)) \quad \text { on }[R, \infty) \\
w(R)=1 \\
\sup \{r \mid w(r)>0\}=q<\infty,
\end{gathered}
$$

where $\mu$ and $R$ are given, and $q$ is determined uniquely by $\mu$ and $R$, so that we write $q=G_{R}(\mu)$. We must first show that as $\mu$ tends to infinity, so does $q$.

LEMMA 3.2. $\lim _{\mu \rightarrow \infty} G_{R}(\mu)=\infty$.

Proof. We seek $y(r)$ to solve:

$$
\begin{aligned}
y^{\prime \prime}+\frac{1}{r} y^{\prime} & =\frac{1}{\mu} f(1) \quad R<r<\hat{R} \\
y(R) & =1, \quad y(\hat{R})=0, \quad y^{\prime}(\hat{R})=0 .
\end{aligned}
$$


Here $\hat{R}$ is determined by the requirement that (3.2) has a solution, so that $\hat{R}=\hat{R}(\mu)$. One can integrate (3.2) explicitly to obtain:

$$
\mu=\frac{f(1)}{2}\left[\frac{R^{2}-\hat{R}^{2}}{4}+\hat{R}^{2} \log \left(\frac{\hat{R}}{R}\right)\right],
$$

and that $y^{\prime}<0$ on $(R, \hat{R})$.

As $\mu$ tends to infinity, it is clear that $R$ must become infinite. We must now show that $G_{R}(\mu) \geq \hat{R}(\mu)$. But this is clear, since

$$
\Delta y-\frac{1}{\mu} f(y) \geq \Delta w-\frac{1}{\mu} f(w) .
$$

If $G_{R}(\mu)<\hat{R}(\mu)$, then $w(R)=y(R)=1$ and $w(\hat{R})=y(\hat{R})=0$. This implies that $w(r) \geq y(r)$, a contradiction.

To show that the free boundaries are asymptotic to a family of circles, it is necessary to show that the highest order term of $G_{R}(\mu)$ as $\mu$ tends to infinity does not depend on $R$. To make this idea precise, we must make the following definition.

Definition. A family of functions $H_{R}(x)$ parametrized by $R$ is asymptotically independent of $R$ as $x$ tends to infinity if:

$$
\lim _{x \rightarrow \infty} \frac{H_{R_{1}}(x)}{H_{R_{2}}(x)}=1
$$

for any $R_{1}, R_{2}$ in the parameter set.

Thus we seek to show that $G_{R}(\mu)$ is asymptotically independent of $R$ as $\mu$ tends to infinity. To do this, we must rewrite (3.1) as an integral equation:

$$
w(r)=\int_{r}^{q} \int_{t}^{q} \frac{\rho}{\mu t} f(w(\rho)) d \rho d t .
$$

Changing the order of integration:

$$
\begin{aligned}
w(r) & =\int_{r}^{q} \int_{r}^{q} \frac{\rho}{\mu t} f(w(\rho)) d t d \rho \\
& =\int_{r}^{q} \rho \log \rho \frac{f(w(\rho))}{\mu} d \rho-\int_{r}^{q} \rho \log r \frac{f(w(\rho))}{\mu} d \rho .
\end{aligned}
$$

Hence,

$$
\mu=\int_{R}^{q} \rho \log \rho f(w(\rho)) d \rho-\log R \int_{R}^{q} \rho f(w(\rho)) d \rho,
$$

since $w(R)=1$.

We will need to use $q$ as a parameter, therefore it is necessary to show that two solutions of (3.1) with the same value of $q$ and of $R$ but possibly different values of $\mu$ are in fact identical. 
LEMMA 3.3. Suppose that $w_{1}(r)$ and $w_{2}(r)$ solve (3.1) with the same $R$, but with $\mu_{1}$ and $\mu_{2}$ not necessarily equal, and that the corresponding $q$ 's are equal. Then $\mu_{1}=\mu_{2}$ and $w_{1}(r)=w_{2}(r)$ for all $r>R$.

Proof. First we must show that if $w(r)$ solves (3.1), then $w^{\prime}(r)<0$ on $(R, q)$ and $w^{\prime \prime}(r)>0$ on $(R, q)$. Rewrite (3.1) as

$$
\frac{d}{d r}\left(r w^{\prime}(r)\right)=\frac{r}{\mu} f(w)
$$

it is clear that $r w^{\prime}(r)$ increases to a value of zero at $r=q$. Hence $w^{\prime}(r)<0$ on $(R, q)$. The fact that $w^{\prime \prime}(r)$ is positive on this interval follows immediately from (3.1).

Now, we may assume that $\mu_{1} \leq \mu_{1}$. It follows that $w_{2}(r) \geq w_{1}(r)$ for all $r$ from Lemma 3.1. Suppose $w_{2}(r)>w_{2}(r)$ for some $r \in(R, q)$. Define $w_{\delta}(r) \equiv w_{1}(r-\delta)$, so that

$$
\begin{aligned}
& w_{\delta}^{\prime \prime}+\frac{1}{r-\delta} w_{\delta}^{\prime}=\frac{1}{\mu_{1}} f\left(w_{\delta}\right) \\
& w_{\delta}(q+\delta)=0 \\
& w_{\delta}(R+\delta)=1 .
\end{aligned}
$$

For $\delta$ sufficiently small we must have $w_{2}(r)>w_{\delta}(r)$ for some $r \in(R+$ $\delta, q)$. However,

$$
\begin{gathered}
w_{\delta}^{\prime \prime}+\frac{1}{r} w_{\delta}^{\prime}-\frac{1}{\mu_{2}} f\left(w_{\delta}\right) \geq 0 \\
1=w_{\delta}(R+\delta)>w_{2}(R+\delta), \text { and } w_{\delta}(q)>w_{2}(q)=0 .
\end{gathered}
$$

It therefore follows that $w_{\delta}(r)>w_{2}(r)$ on $(R+\delta, q)$, which is a contradiction. Hence $w_{1}(r)=w_{2}(r)$ for all $r \in(R, q)$, so that $\mu_{1}=\mu_{2}$.

It follows from this lemma that solutions to (3.1) may be parametrized by $R$ and $q$, and that for fixed $R$ the function $q=G_{R}(\mu)$ has an inverse. From (3.3), this inverse can be expressed as:

$$
\begin{aligned}
\mu= & G_{R}^{-1}(q)=\int_{R}^{q} \rho \log \rho f(w(\rho ; q, R)) d \rho \\
& -\log R \int_{R}^{q} \rho f(w(\rho ; q, R)) d \rho,
\end{aligned}
$$

where we write $w(\rho ; q, R)$ to indicate the dependence of $w$ on the parameters $q$ and $R$. I will sometimes use $\mu(q, R)$ instead of $G_{R}^{-1}(q)$. They are the same. We will see from this integral representation that $G_{R}^{-1}(q)$ is asymptotically independent of $R$ for large $q$, and then use an inverse lemma to show that $G_{R}(\mu)$ is asymptotically independent of $R$ for large $\mu$. 
LEMMA 3.4. For all $r \geq R$, with $R$ fixed, $\lim _{q \rightarrow \infty} w(r ; q, R)=1$, and $\lim _{q \rightarrow \infty} w^{\prime}(r ; q, R)=0$.

Proof. This is obtained by a shooting argument. We first need a lower bound on $w^{\prime}(R ; q, R)$ independent of $q$ if $q$ is sufficiently large. Let $b(r)$ solve

$$
b^{\prime \prime}+\frac{1}{r} b^{\prime}=1, \quad b(R)=1, \quad b(2 R)=0 .
$$

From Lemma 3.2, as $q$ tends to infinity, so does $\mu$. Therefore, for $q>2 R$ sufficiently large, we have:

$$
\Delta w(r ; q, R)<\Delta b, \quad w(R ; q, R)=b(R), \quad w(2 R ; q, R)>b(2 R),
$$

hence $w^{\prime}(R ; q, R)>b^{\prime}(R)$ for $q$ sufficiently large, the desired bound.

For any subsequence $q_{i}$ tending to infinity, we can, by taking a subsequence, assume that $\lim _{i \rightarrow \infty} w^{\prime}(R ; q, R)=L$ with $b^{\prime}(R) \leq L \leq 0$. Let $z(r)$ be that function which solves:

$$
z^{\prime \prime}+\frac{1}{r} z^{\prime}=0, \quad z(R)=1, \quad z^{\prime}(R)=L .
$$

By the theorem on continuous dependence of solutions of O.D.E.'s on their parameters, $w\left(r ; q_{i}, R\right)$ approaches $z(r)$ almost uniformly on the domain of existence of $z(r)$, call it $\left[R, R_{1}\right)$. It remains to be shown that $R_{1}=+\infty$. From the almost uniform convergence of $w\left(r ; q_{i}, R\right)$ to $z(r)$, it follows that $z^{\prime}(r) \leq 0$ and $z(r)>0$ on $\left[R, R_{1}\right)$. Hence $z^{\prime \prime}(r) \geq 0$, so that $L \leq z^{\prime}(r) \leq 0$ for all $r \in\left[R, R_{1}\right)$. But this implies a bound on $z^{\prime \prime}(r)$ as well. Thus, by well-known existence theorems, $R_{1}=+\infty$. We therefore have $z(r)$ satisfying:

$$
z^{\prime \prime}(r)+\frac{1}{r} z^{\prime}(r)=0, \quad z(R)=1, \quad z(r) \geq 0, \quad z^{\prime}(r) \leq 0
$$

for $R<r<+\infty$. It is easy to see by an explicit integration that $z(r) \equiv 1$ is the only solution. This implies the desired result.

LEMMA 3.5.

$$
\lim _{q \rightarrow \infty} \frac{\int_{R}^{q} \rho f(w(\rho ; q, R)) d \rho}{\int_{R}^{q} \rho \log \rho f(w(\rho ; q, R)) d \rho}=0 .
$$

Proof. Integrating by parts:

$$
\int_{R}^{q} \rho f(w(\rho ; q, R)) d \rho=\left(-R w^{\prime}(R ; q, R)\right) \mu(q, R,
$$


and

$$
\int_{R}^{q} \rho \log \rho f(w(\rho ; q, R)) d \rho=\left(1-R \log R w^{\prime}(R ; q, R)\right) \mu(q, R) .
$$

Since $R$ is fixed, the result follows immediately from Lemma 3.4.

LEMMA 3.6. If $R_{1}>R$, then $\mu\left(q, R_{1}\right)<\mu(q, R)$.

Proof. Suppose that $\mu\left(q, R_{1}\right) \geq \mu(q, R)$. Define $w_{\delta}(r)=$ $w(r-\delta ; q, R)$, with $\delta>0$, so that

$$
w_{\delta}^{\prime \prime}(r)+\frac{1}{r-\delta} w_{\delta}^{\prime}(r)=\frac{1}{\mu(q, R)} f\left(w_{\delta}(r)\right),
$$

implying that

$$
w_{\delta}^{\prime \prime}(r)+\frac{1}{r} w_{\delta}^{\prime}(r) \geq \frac{1}{\mu(q, R)} f\left(w_{\delta}(r)\right) \geq \frac{1}{\mu\left(q, R_{1}\right)} f\left(w_{\delta}(r)\right),
$$

the last inequality by our assumption. Now set $\delta$ to be $R_{1}-R$. We have:

$$
w_{\delta}\left(R_{1}\right)=1=w\left(R_{1} ; q, R_{1}\right)
$$

amd

$$
w_{\delta}\left(q+R_{1}-R\right)=0=w\left(q+\delta ; q, R_{1}\right) .
$$

By the comparison principle for quasi-linear elliptic operators, we must have

$$
w_{\delta}(r) \leq w\left(r ; q, R_{1}\right)
$$

But this is contradicted at $r=q$.

LEMMA 3.7. If $R<R_{1}<r$, then

$$
w\left(r ; q, R_{1}\right) \geq w(r ; q, R) .
$$

Proof. Define $w_{\varepsilon}(r)=w\left(r-\varepsilon ; q, R_{1}\right)$, for $\varepsilon>0$. Then:

$$
w_{\varepsilon}^{\prime \prime}+\frac{1}{r} w_{\varepsilon}^{\prime} \geq \frac{1}{\mu\left(q, R_{1}\right)} f\left(w_{\varepsilon}\right) \geq \frac{1}{\mu(q, R)} f\left(w_{\varepsilon}\right) \text {, }
$$

the last inequality from Lemma 3.6. Suppose for some $\hat{r} \in\left(R_{1}+\varepsilon, q\right)$, we have

$$
w_{\varepsilon}(\hat{r}) \leq w(\hat{r} ; q, R) .
$$

Since $w_{\varepsilon}(q+\varepsilon)=w(q+\varepsilon ; q+\varepsilon, R)=0$, it follows from the comparison principle that

$$
w_{\varepsilon}(r) \leq w(r ; q, R)
$$


for $r \in(\hat{r}, q+\varepsilon)$. However, this is violated at $r=q$. We therefore conclude that

$$
w_{\varepsilon}(r)>w(r ; q, R)
$$

for all $r \in(R+\varepsilon, q)$. Now let $\varepsilon$ tend to zero to obtain the desired result.

LEMMA 3.8. The family of functions $\mu=G_{R}^{-1}(q)$ is asymptotically independent of $R$ as $q$ tends to infinity.

Proof. From Lemma 3.5 and equation (3.4), it follows that to prove asymptotic independence of $G_{R}^{-1}(q)$, we must show that $\int_{R}^{q} \rho \log \rho f(w(\rho ; q, R)) d \rho$ is asymptotically independent of $R$ for large $q$. We must examine the quotient

$$
\frac{\int_{R}^{q} \rho \log \rho f(w(\rho ; q, R)) d \rho}{\int_{R_{1}}^{q} \rho \log \rho f\left(w\left(\rho ; q, R_{1}\right)\right) d \rho}
$$

with $R_{1}$ assumed to be larger than $R$. Integrating by parts, this is:

$$
\frac{\mu(q, R)\left[1-R \log R w^{\prime}(R ; q, R)\right]}{\mu\left(q, R_{1}\right)\left[1-R_{1} \log R_{1} w^{\prime}\left(R_{1} ; q, R_{1}\right)\right]} .
$$

From Lemma 3.4, the quantities in brackets in both the numerator and denominator approach 1. Therefore, using Lemma 3.6,

$$
\liminf _{q \rightarrow \infty} \frac{\int_{R}^{q} \rho \log \rho f(w(\rho ; q, R)) d \rho}{\int_{R_{1}}^{q} \rho \log \rho f\left(w\left(\rho ; q, R_{1}\right)\right) d \rho} \geq 1
$$

Now, write (3.5) as

$$
\frac{\int_{R}^{R_{1}} \rho \log \rho f(w(\rho ; q, R)) d \rho}{\int_{R_{1}}^{q} \rho \log \rho f\left(w\left(\rho ; q, R_{1}\right)\right) d \rho}+\frac{\int_{R_{1}}^{q} \rho \log \rho f(w(\rho ; q, R)) d \rho}{\int_{R_{1}}^{q} \rho \log \rho f\left(w\left(\rho ; q, R_{1}\right)\right) d \rho} .
$$

By Lemma 3.7 and the monotonicity of $f$, the second fraction in (3.6) is bounded from above by 1 . We must therefore prove that the first fraction in (3.6) goes to zero. But this is clear, since the numerator is bounded by $\left|\int_{R}^{R_{1}} \rho \log \rho f(1) d \rho\right|$, and the denominator grows like $\mu\left(q, R_{1}\right)$. By this argument,

$$
\underset{q \rightarrow \infty}{\limsup } \frac{\int_{R}^{q} \rho \log \rho f(w(\rho ; q, R)) d \rho}{\int_{R_{1}}^{q} \rho \log \rho f\left(w\left(\rho ; q, R_{1}\right)\right) d \rho} \leq 1 .
$$

Hence

$$
\lim _{q \rightarrow \infty} \frac{\int_{R}^{q} \rho \log \rho f(w(\rho ; q, R)) d \rho}{\int_{R_{1}}^{q} \rho \log \rho f\left(w\left(\rho ; q, R_{1}\right)\right) d \rho}=1
$$


We therefore conclude that

$$
\lim _{q \rightarrow \infty} \frac{G_{R}^{-1}(q)}{G_{R_{1}}^{-1}(q)}=1,
$$

i.e. that $G_{R}^{-1}(q)$ is asymptotically independent of $R$ as $q$ tends to $\infty$.

We must now use the asymptotic independence of $G_{R}^{-1}(q)$ to establish the asymptotic independence of $G_{R}(\mu)$. One might think that this follows immediately; that if $y=g_{R}(x)$ is insensitive to the value of $R$ as $x$ (and $y$ ) tend to infinity, then $x=g_{R}^{-1}(y)$ should exhibit the same property. A simple counter-example is $g_{R}(x)=R+\log x$, so that $g_{R}^{-1}(y)=e^{y-R}$. Here $g_{R}(x)$ is clearly asymptotically independent of $R$, and just as clearly we have asymptotic dependence on $R$ of $g_{R}^{-1}(y)$. We must therefore derive conditions which will imply the asymptotic independence of the family of inverse functions and shown that these conditions are satisfied in our particular case. The following lemma is more tractable for $x$ tending to infinity than for $x$ tending to zero, which is why we use the parameter $\mu$ rather than $\lambda$.

LEMMA 3.9. Suppose that the family of functions $F_{R}(x)$, defined for $x>R$, satisfies:

(a) $\lim _{x \rightarrow \infty} F_{R}(x)=\infty$ for all $R$

(b) $F_{R}(x)$ is positive and monotonically increasing in $x$ for fixed $R$

(c) $\left\{F_{R}(x)\right\}$ is asymptotically independent of $R$ as $x$ tends to infinity

(d) $\left(F_{R}(x+\Delta x)-F_{R}(x)\right) / \Delta x \geq F_{R}(x) / x$ for all $x, R$, and $\Delta x$ for which these quantities are defined.

Then the family $\left\{F_{R}^{-1}(y)\right\}$ is asymptotically independent of $R$ as $y$ tends to infinity. (Notice that no differentiability assumptions are made on $F_{R}(x)$.)

Proof. From condition (d), we have:

$$
\left|\frac{F_{R}(x+\Delta x)-F_{R}(x)}{F_{R}(x)}\right| \geq\left|\frac{\Delta x}{x}\right|
$$

for all $x, R$, and $\Delta x$ for which these quantities are defined, including $\Delta x=0$. Fix $R$ and $R_{1}$, with $R_{1}>R$. Pick $y>\max \left(F_{R_{1}}\left(R_{1}\right), F_{R}(R)\right)$, and let $x=F_{R_{1}}^{-1}(y)$. Let $\Delta x=F_{R}^{-1}\left(F_{R_{1}}(x)\right)-x$. (The choice of $y$ ensures that $F_{R_{1}}(x)$ is in the domain of $\left.F_{R}^{-1}\right)$. Putting these particular values of $x$ and $\Delta x$ into (3.7) we obtain:

$$
\left|\frac{F_{R_{1}}(x)-F_{R}(x)}{F_{R}(x)}\right| \geq\left|\frac{F_{R}^{-1}(y)-F_{R_{1}}^{-1}(y)}{F_{R_{1}}^{-1}(y)}\right| .
$$


As $y$ tends to infinity, so does $x=F_{R_{1}}^{-1}(y)$ from assumption (a). Therefore, from assumption (c), the left hand side of (3.8) must approach zero. We conclude that:

$$
\lim _{y \rightarrow \infty} \frac{F_{R}^{-1}(y)}{F_{R_{1}}^{-1}(y)}=1
$$

establishing the lemma.

Note. Condition (d) can clearly be weakened by inserting a multiplicative constant depending on $R$.

LEMMA 3.10. $\mu(q, R) /(q-R)^{2}$ is monotonically increasing in $q$ for fixed $R$.

Proof. Take $\Delta q>0$. Define $s(r ; q)$ to be $\mu(R, q) w(r ; q, R)$, so that:

$$
s^{\prime \prime}+\frac{1}{r} s^{\prime}=f\left(\frac{s}{\mu}\right)
$$

and consider the inhomogeneous scaling

$$
z_{\beta}(r)=\frac{\mu(q, R)}{\mu(q+\Delta q, R)} s(R+(r-R) \beta ; q+\Delta q) .
$$

We have

$$
\begin{aligned}
\frac{1}{\beta^{2}} \frac{\mu(q+\Delta q, R)}{\mu(q, R)} & z_{\beta}^{\prime \prime}+\frac{1}{\beta} \frac{\mu(q+\Delta q, R)}{\mu(q, R)} \frac{1}{R+(r-R) \beta} z_{\beta}^{\prime} \\
= & f\left(\frac{z_{\beta}}{\mu(q, R)}\right) .
\end{aligned}
$$

Set

$$
\beta=\sqrt{\frac{\mu(q+\Delta q, R)}{\mu(q, R)}},
$$

so that

$$
z_{\beta}^{\prime \prime}+\frac{1}{R \sqrt{\mu(q, R) / \mu(q+\Delta q, R)}+(r-R)} z_{\beta}^{\prime}=f\left(\frac{z_{\beta}}{\mu(q, R)}\right) .
$$

We have that $\mu(q, R)$ is increasing in $q$ for fixed $R$ from Lemma 3.1, so that

$$
z_{\beta}^{\prime \prime}+\frac{1}{r} z_{\beta}^{\prime} \geq f\left(\frac{z_{\beta}}{\mu(q, R)}\right)
$$


and

$$
z_{\beta}(R)=\mu(q, R), z_{\beta}(q+\Delta q)=0 .
$$

Using the comparison principle for quasi-linear elliptic operators, we conclude that

$$
z_{\beta}(r) \leq s(r, q) .
$$

Now, $z_{\beta}(r)$ is first zero at

$$
\hat{r}=R+\sqrt{\frac{\mu(q, R)}{\mu(q+\Delta q, R)}}(q+\Delta q-R) \leq q,
$$

the last inequality since $s(\hat{r}, q) \geq 0$. Hence

$$
\frac{\mu(q, R)}{(q-R)^{2}} \leq \frac{\mu(q+\Delta q, R)}{(q+\Delta q-R)^{2}},
$$

as desired.

THEOREM 3.11. The family of function $\left\{G_{R}(\mu)\right\}$ is asymptotically independent of $R$ as $\mu$ tends to infinity.

Proof. We must verify that each of the conditions of Lemma 3.9 hold for the family $\left\{G_{R}^{-1}(q)\right\}$. Condition (a) follows from Lemma 3.2. Condition (b) comes from Lemma 3.1. Condition (c) is proven in Lemma 3.8. It remains for us to prove that condition (d) holds. First assume that $\Delta q>0$. From Lemma 3.10, we have

$$
\frac{\mu(q+\Delta q, R)}{(q+\Delta q-R)} \geq \frac{\mu(q, R)}{(q-R)^{2}},
$$

thus

$$
\frac{\mu(q+\Delta q, R)}{q+\Delta q-R}>\frac{\mu(q, R)}{q-R}
$$

implying

$$
(q-R)(\mu(q+\Delta q, R)-\mu(q, R))>\Delta q \mu(q, R) .
$$

That is,

$$
\frac{G_{R}^{-1}(q+\Delta q)-G_{R}^{-1}(q)}{\Delta q}>\frac{G_{R}^{-1}(q)}{q-R}>\frac{G_{R}^{-1}(q)}{q} .
$$

The proof for $\Delta q<0$ is similar. This establishes condition (d) of Lemma 3.9 , proving the theorem. 
THEOREM 3.12. Let $\Omega \subseteq \mathbf{R}^{\perp}$ be a bounded open set with $C^{2+\alpha}$ boundary. Let $v_{\lambda}(x, y)$ solve Problem $A$, and let $\Gamma_{\lambda}$ be the corresponding free boundary. Then as $\lambda$ tends to zero, $\Gamma_{\lambda}$ is asymptotic to a family of concentric circles with center in $\Omega$. Moreover, the rate of growth of these circles depends only on $\lambda$, not on $\Omega$. To be precise, if

$$
d\left(\Gamma_{\lambda}, p\right)=\inf _{q \in \Gamma_{\lambda}} \operatorname{dist}(p, q) \text { and } d_{1}\left(\Gamma_{\lambda}, p\right)=\sup _{q \in \Gamma_{\lambda}} \operatorname{dist}(p, q),
$$

for some fixed $p \in \Omega$, then

$$
\lim _{\lambda \rightarrow 0} \frac{d\left(\Gamma_{\lambda}, p\right)}{d_{1}\left(\Gamma_{\lambda}, p\right)}=1
$$

Proof. Let $B_{1}(p)$ be a ball of radius $r_{1}$ contained in $\Omega$ with center $p$, and let $B_{2}(p)$ be a ball of radius $r_{2}$ containing $\Omega$. From Lemma 3.1, $\Gamma_{\lambda}$ is contained in the annulus with center $p$, inner radius $G_{r_{1}}(\mu)$ and outer radius $G_{r_{2}}(\mu)$ (here $\left.\mu=1 / \lambda\right)$. From Theorem 3.11, we have

$$
\lim _{\mu \rightarrow \infty} \frac{G_{r_{1}}(\mu)}{G_{r_{2}}(\mu)}=1,
$$

completing the proof.

4. Asymptotic behavior of Problem B as $c$ approaches $\infty$. Proving the result analogous to Theorem 3.12 in the case of problem B involves much the same chain of logic as in $\$ 3$. I will therefore omit proofs when they are obvious modifications of those lemmas in $\S 3$. We will need an assumption of $f(t)$ in addition to assumptions (2.1), (a)-(d).

(e) $\lim _{t \rightarrow \infty} f(t) / t=0$.

LEMMA 4.1. Let $\Omega_{1} \subset \Omega_{2}$ be bounded open sets and let $c_{2} \geq c_{1}$. Let $v_{i}$ solve Problem $B$ corresponding to $\Omega_{i}$. Then $v_{2}(x) \geq v_{1}(x)$ everywhere.

Proof. See Lemma 3.1.

The radially symmetric functions $z(r)$ for problem B solve:

$$
\begin{aligned}
z^{\prime \prime}(r)+\frac{1}{r} z(r) & =f(z) \quad \text { on }(R, \infty) \\
z(R) & =c>0, \quad \sup \{r \mid z(r)>0\}=q<\infty .
\end{aligned}
$$

Here $R$ and $c$ are given, and $q$ is determined uniquely by $R$ and $c$, so we write $q=H_{R}(c)$. We must show that $c$ tends to infinity, so does $q$. 
LEMMA 4.2. $\lim _{c \rightarrow \infty} H_{R}(c)=\infty$.

Proof. Let $\tilde{y}(r)$ solve:

$$
\begin{aligned}
\tilde{y}^{\prime \prime}+\frac{1}{r} \tilde{y}^{\prime} & =f(c) \\
\tilde{y}(R) & =c, \quad \tilde{y}(\hat{R})=0, \quad \tilde{y}^{\prime}(\hat{R})=0 .
\end{aligned}
$$

Integrating (4.2) explicitly, we get

$$
0=\frac{\hat{R}^{2}-R^{2}}{4}+\frac{\hat{R}}{2} \log \left(\frac{R}{\hat{R}}\right)+\frac{c}{f(c)} .
$$

By assumption (e), $c / f(c) \rightarrow \infty$, therefore we must have $\lim _{c \rightarrow \infty} \hat{R}(c)=\infty$. As in Lemma 3.2, $H_{R}(c) \geq \hat{R}(c)$, proving the lemma.

LEMMA 4.3. Suppose that $z_{1}(r)$ and $z_{2}(r)$ solve (4.1) with the same value of $q$, but possibly differing $R_{1}, R_{2}$ and $c_{1}, c_{2}$. Then $z_{1}(r)=z_{2}(r)$ where they are both defined.

Proof. As in Lemma 3.3, $z^{\prime}(r)<0$ and $z^{\prime \prime}(r)>0$ on $(R, q)$. Let $z_{\delta}(r)=z_{1}(r-\delta)$ for $\delta>0$. Then

$$
z_{\delta}^{\prime \prime}+\frac{1}{r} z_{\delta}^{\prime} \geq f\left(z_{\delta}\right) .
$$

If $z_{\delta}(\hat{r})<z_{2}(\hat{r})$ for $\max \left(R_{1}, R_{2}\right)<\hat{r}<q$, then it follows from the comparison principle that $z_{\delta}(q)<z_{2}(q)$, since $z_{\delta}(q+\delta)=z_{2}(q+\delta)$. But this is a contradiction. Hence $z_{\delta}(r) \geq z_{2}(r)$ on their domain of mutual definition. Letting $\delta$ tend to zero, we obtain $z_{1}(r) \geq z_{1}(r)$ for $\max \left(R_{1}, R_{2}\right)<r<q$. But since $z_{1}$ and $z_{2}$ are not distinguished, we obtain $z_{1}(r)=z_{2}(r)$.

Note. This result is much stronger that Lemma 3.3, the analogous result for Problem A. Using Lemma 4.3, we may parametrize solutions to (4.1) by $q$ and $R$. The analog in equation (3.4) is:

(4.3) $c=H_{R}^{-1}(q)$

$$
=\int_{R}^{q} \rho \log \rho f(z(\rho ; q, R)) d \rho-\log R \int_{R}^{q} \rho f(z(\rho ; q, R)) d \rho,
$$

where I will sometimes write $c(q, R)$ for $H_{R}^{-1}(q)$. Of course $c(q, R)=$ $z(R ; q, R)$.

LEMMA 4.4. For all $r \geq R$,

$$
\lim _{q \rightarrow \infty} \frac{z(r ; q, R)}{c(q, R)}=1, \quad \text { and } \quad \lim _{q \rightarrow \infty} \frac{z^{\prime}(r ; q, R)}{c(q, R)}=0 .
$$


Proof. Let $y(r)=z(r ; q, R) / c(q, R)$. We have

$$
y^{\prime \prime}+\frac{1}{r} y^{\prime}=\frac{1}{c} f(c y), \quad y(R)=1 .
$$

From assumption e) on the function $f$, we have that the right hand side of (4.4) approaches zero as $c$ tends to infinity. This is enough to ensure that the shooting argument of Lemma 3.4 works.

LEMMA 4.5.

$$
\lim _{q \rightarrow \infty} \frac{\int_{R}^{q} \rho f(z(\rho ; q, R)) d \rho}{\int_{R}^{q} \rho \log \rho f(z(\rho ; q, R)) d \rho}=0 .
$$

Proof. Integrating by parts, this fraction is:

$$
\frac{-R z^{\prime}(R ; q, R)}{c(q, R)-R \log R z^{\prime}(R ; q, R)},
$$

from which the result follows by Lemma 4.4.

LEMMA 4.6. $H_{R}^{-1}(q)$ is asymptotically independent of $R$ as $q$ tends to infinity.

Proof. From Lemma 4.5 and equation (4.3), it follows that to prove asymptotic independence of $H_{R}^{-1}(q)$, we must show that $\int_{R}^{q} \rho \log f(z(\rho ; q, R)) d \rho$ is asymptotically independent of $R$ for large $q$. This is an easier task than proving Lemma 3.8, because of the strength of Lemma 4.3. We must examine the quotient:

$$
\frac{\int_{R}^{q} \rho \log \rho f(z(\rho ; q, R)) d \rho}{\int_{R}^{R_{1}} \rho \log \rho f(z(\rho ; q, R)) d \rho} .
$$

Write this as

$$
\frac{\frac{1}{f(c(q, R))} \int_{R}^{q} \rho \log \rho f(z(\rho ; q, R)) d \rho}{\int_{R}^{R_{1}} \rho \log \rho \frac{f(z(\rho ; q, R))}{f(c(q, R))} d \rho} .
$$

The denominator of (4.5) is bounded, by the monotonicity of $z$ and of $f$. The numerator of $(4.5)$ can be written as:

$$
\frac{c(q, R)}{f(c(q, R))}\left[1-\frac{z^{\prime}(R ; q, R)}{c(q, R)} R \log R\right],
$$

after an integration by parts. By Lemma 4.4, the quantity in brackets 
approaches 1 as $q$ tends to infinity. By assumption (e) on $f$;

$$
\lim _{q \rightarrow \infty} \frac{c(q, R)}{f(c(q, R))}=\infty
$$

since $\lim _{q \rightarrow \infty} c(q, R)=\infty$. Therefore the numerator of (4.5) approaches infinity, so that:

$$
\lim _{q \rightarrow \infty}\left(\frac{\int_{R}^{q} \rho \log \rho f(z(\rho ; q, R)) d \rho}{\int_{R}^{R_{1}} \rho \log \rho f(z(\rho ; q, R)) d \rho}\right)=\infty .
$$

Therefore, for $R_{1}>R$,

$$
\begin{aligned}
& \lim _{q \rightarrow \infty}\left(\frac{\int_{R_{1}}^{q} \rho \log \rho f\left(z\left(\rho ; q, R_{1}\right)\right) d \rho}{\int_{R}^{q} \rho \log \rho f(z(\rho ; q, R)) d \rho}\right) \\
& \quad= \lim _{q \rightarrow \infty}\left(\frac{\int_{R_{1}}^{q} \rho \log \rho f(z(\rho ; q, R)) d \rho}{\int_{R}^{R_{1}} \rho \log \rho f(z(\rho ; q, R)) d \rho+\int_{R_{1}}^{q} \rho \log \rho f(z(\rho ; q, R)) d \rho}\right) \\
& \quad=1 .
\end{aligned}
$$

We have used the fact that $z(\rho ; q, R)=z\left(\rho ; q, R_{1}\right)$ for $r>R_{1}$. This demonstrates the asymptotic independence of $\int_{R}^{q} \rho \log \rho f(z(\rho ; q, R)) d \rho$ and hence of $H_{R}^{-1}(q)$.

To show that $H_{R}(c)$ is asymptotically independent of $R$ for large $c$, we must verify that the hypotheses of Lemma 3.9 hold. This is done by proving a result analogous to Lemma 3.10.

LEMMA 4.7. $c(q, R) /(q-R)^{2}$ is monotonically increasing as a function of $q$ for fixed $R$.

Proof. We use an inhomogeneous scaling which differs from that of Lemma 3.10. Take $\Delta q>0$ and let:

$$
w_{\alpha}(r)=\alpha z\left(R+(r-R)\left(\frac{q+\Delta q-R}{q-R}\right) ; q+\Delta q, R\right),
$$

so that $w_{\alpha}(q)=0, w_{\alpha}^{\prime}(q)=0$. We have that:

$$
\begin{aligned}
& \frac{1}{\alpha}\left(\frac{q-R}{q+\Delta q-R}\right)^{2} w_{\alpha}^{\prime \prime} \\
& \quad+\frac{1}{R+(r-R)((q+\Delta q-R) /(q-R))}\left(\frac{1}{\alpha}\right)\left(\frac{q-R}{q+\Delta q-R}\right) w_{\alpha}^{\prime} \\
& \quad=f\left(\frac{1}{\alpha} w_{\alpha}\right) .
\end{aligned}
$$


Now choose $\alpha=((q-R) /(q+\Delta q-R))^{2}$, so that:

$$
w_{\alpha}^{\prime \prime}+\frac{1}{R((q-R) /(q+\Delta q-R))+(r-R)} w_{\alpha}^{\prime}=f\left(\frac{1}{\alpha} w_{\alpha}\right) \geq f\left(w_{\alpha}\right) .
$$

Since $w_{\alpha}^{\prime} \leq 0$, it follows that:

$$
w_{\alpha}^{\prime \prime}+\frac{1}{r} w_{\alpha}^{\prime} \geq f\left(w_{\alpha}\right)
$$

I claim that $w_{\alpha}(r) \geq z(r ; q, R)$. Indeed, if this were not the case, we can let $w_{\delta}(r)=w_{\alpha}(r-\delta)$, so that

$$
w_{\delta}^{\prime \prime}+\frac{1}{r} w_{\delta}^{\prime} \geq f\left(w_{\delta}\right) .
$$

If $w_{\delta}(\hat{r})=z(\hat{r} ; q, R)$ for some $\hat{r}<q$, then we can use the comparison principle to say that $w_{\delta}(q) \leq z(q ; q, R)=0$, since $w_{\delta}(q+\delta)=$ $z(q+\delta ; q, R)$. But this is a contradiction, because $w_{\delta}(q)>0$. Therefore $w_{\delta}(r)>z(r ; q, R)$ for $R+\delta \leq r<q$. Now let $\delta \rightarrow 0$ to conclude that $w_{\alpha}(r) \geq z(r ; q, R)$. At $r=R$ this implies:

$$
\left(\frac{q-R}{q+\Delta q-R}\right)^{2} z(R ; q+\Delta q, R) \geq z(R ; q, R)
$$

yielding the desired result.

THEOREM 4.8. The family of functions $\left\{H_{R}(c)\right\}$ is asymptotically independent of $R$ as $c$ tends to infinity.

Proof. Same proof as Theorem 3.11.

TheOREM 4.9. Let $\Omega \subseteq \mathbf{R}^{2}$ be a bounded open set with $C^{2+\alpha}$ boundary. Let $w_{c}(x, y)$ solve Problem $\mathrm{B}$, and let $\Gamma_{c}$ be the corresponding free boundary. Then as $c \rightarrow \infty, \Gamma_{c}$ is asymptotic to a family of concentric circles with center in $\Omega$. The rate of growth of these circles does not depend on $\Omega$.

Proof. Same as the proof of Theorem 3.12.

\section{REFERENCES}

[1] R. Aris, The Mathematical Theory of Diffusion and Reaction in Permeable Catalysts, vol. I, Clarendon Press, Oxford, (1975).

[2] C. M. Brauner and B. Nicolaenko, Free boundary problems as singular limits of non-linear eigenvalue problems, in Free Boundary Problems, vol. II, proceedings of a seminar held in Pavia, 1979. 
[3] L. A. Caffarelli and J. Spruck, Convexity properties of solutions to some classic variational problems, Comm. in P. D. E., 7 (11) (1982), 1337-1379.

[4] A. Friedman, Variational Principles and Free-Boundary Problems, John Wiley \& Sons, New York, 1982.

[5] A. Friedman and D. Phillips, The free boundary of a semi-linear elliptic equation, Trans. Amer. Math. Soc., 282 no. 1, (1984), 153-182.

[6] D. Gilbarg and M. S. Trudinger, Elliptic Partial Differential Equations of Second Order, Springer-Verlag, Berlin, 1977.

[7] D. Phillips, Hausdorff measure estimates of a free boundary for a minimum problem, Comm. in P. D. E., 8 (13), 1983, 1409-1454.

[8] _ A minimization problem and the regularity of solutions in the presence of a free boundary, Indiana U. Math. J., 32 no. 1, (1983), 1-17.

[9] T. Vogel, A free-boundary problem arising from a galvanizing process, SIAM J. of Math Analysis, 16 (5), (1985), 970-979.

Received November 2, 1984.

TEXAS A \& M UNIVERSITY

COLlege Station, TX 77843-3368 
